\title{
On Exponentiable Morphisms in Classical Algebra
}

\author{
Maria Manuel Clementino ${ }^{1} \cdot$ Dirk Hofmann $^{2}$. \\ George Janelidze ${ }^{3}$
}

Received: 12 November 2015 / Accepted: 2 February 2016 / Published online: 30 July 2016

(C) Springer Science+Business Media Dordrecht 2016

\begin{abstract}
We study exponentiability of homomorphisms in varieties of universal algebras close to classical ones. After describing an "almost folklore" general result, we present a purely algebraic proof of "étale implies exponentiable", alternative to the topologically motivated proof given in one of our previous papers, in a different context. We prove that only isomorphisms are exponentiable homomorphisms in ideal determined varieties and extend this to ideal determined categories. Finally, we give a complete characterization of exponentiable homomorphisms of semimodules over semirings.
\end{abstract}

Keywords Exponentiable morphism · Taut monad · Subtractive variety · Ideal determined variety $\cdot$ Semimodule

Mathematics Subject Classifications (2010) $18 \mathrm{C} 15 \cdot 18 \mathrm{C} 20 \cdot 08 \mathrm{~A} 62 \cdot 16 \mathrm{Y} 60$

Dedicated to the memory of Horst Herrlich.

Maria Manuel Clementino

mmc@mat.uc.pt

Dirk Hofmann

dirk@ua.pt

George Janelidze

George.Janelidze@uct.ac.za

1 CMUC, Department of Mathematics, University of Coimbra, 3001-501 Coimbra, Portugal

2 CIDMA, Department of Mathematics, University of Aveiro, 3810-193 Aveiro, Portugal

3 Department of Mathematics and Applied Mathematics, University of Cape Town, Rondebosch 7701, Cape Town, South Africa 


\section{Introduction}

Recall that a morphism $f: A \rightarrow B$ in a category $\mathbf{C}$ is said to be exponentiable if the pullback functor $f^{*}:(\mathbf{C} \downarrow B) \rightarrow(\mathbf{C} \downarrow A)$ has a right adjoint. This paper is one of many that attempt to characterize such morphisms in various concrete situations. The categories we are interested in are varieties of universal algebras, which, being close to those of classical algebra, have few exponentiable morphisms. More specifically, the four sections of this paper are devoted to the following four questions respectively:

Question 1.1 What can we say about exponentiability in a variety of universal algebras in general? Since P. T. Johnstone [18] says, after giving an object-wise characterization of cartesian closed varieties, "...the argument of the above proof may be used to characterize the exponentiable objects of $T$-Alg..." one might expect that we aim at a syntactical characterization of exponentiable homomorphisms of algebras. We do not go that far, but only make preliminary remarks, the most important of which is that the exponentiability of $f$ reduces to preservation of finite coproducts by the functor $f^{*}$ - in fact even just to preservation of finite coproducts of objects in $(\mathbf{C} \downarrow B)$ with free domains. This "almost folklore" result is a natural counterpart of Proposition 3.1 in [18], to whose proof the citation above refers.

Question 1.2 After studying, by embedding a variety of algebras in the corresponding category of lax algebras, the exponentiability of the so-called étale homomorphisms (Theorem 5.5 of [4]), one can ask: What is the purely algebraic version of the implication

$$
\text { "étale } \Longrightarrow \text { exponentiable"? }
$$

Surprisingly, the above-mentioned reduction allows us to avoid the advanced machinery of lax algebras used in [4] and prove the implication (1.1) for algebras over any finitary taut monad.

Question 1.3 What are 'sufficiently classical' categories of algebras in which only isomorphisms are exponentiable? We knew for a long time that all semi-abelian categories were such, which includes all varieties of groups with multiple operators in the sense of P. J. Higgins [11] (hence the categories of groups, rings, modules and various types of algebras over rings, crossed modules, etc.). But now we prove that the same is true for all ideal determined categories and in particular for pointed ideal determined varieties of algebras.

Question 1.4 What exactly are exponentiable morphisms of semimodules? This question is answered fully.

Let us also mention an 'unwritten section': It would be devoted to the case where all operations in the given variety are either 0-ary or unary - however, it would be an easy exercise using the fact that the forgetful functor from such a variety to a suitable category of the form $(\mathbf{C} \downarrow$ Sets) preserves coproducts, and the reduction result mentioned in Question 1.1.

Throughout this paper we shall use the following notation:

Unless stated otherwise, $\mathbf{C}$ will denote a variety of (finitary) universal algebras, equipped with the free-forgetful adjunction

$$
(F, U, \eta, \varepsilon): \text { Sets } \rightarrow \mathbf{C}
$$


whose monad will be denoted by $T$.

\section{Exponentiability and Coproducts}

We begin with a theorem that combines rather trivial implications with a special case of a 'folklore' theorem (another special case was used, e.g., in [1], but we could not find a convenient general reference):

Theorem 2.1 The following conditions are equivalent for a morphism $f: A \rightarrow B$ in $\mathbf{C}$ :

(a) $f: A \rightarrow B$ is exponentiable;

(b) the pullback functor $f^{*}:(\mathbf{C} \downarrow B) \rightarrow(\mathbf{C} \downarrow A)$ preserves all small colimits;

(c) the functor $f^{*}$ preserves the initial object and binary coproducts;

(d) the functor $f^{*}$ preserves finite coproducts of objects whose underlying objects in $\mathbf{C}$ are free algebras on one-element sets;

(e) for every set $S$ and every map $g: S \rightarrow U(B)$, the diagram

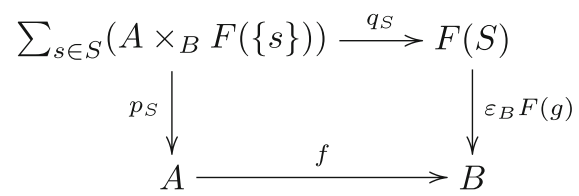

of canonical morphisms, where $p_{S}(a, t)=a$ and $q_{S}(a, t)=t$ for $(a, t) \in A \times{ }_{B} F(\{s\})$ (assuming that $F(\{s\})$ is a subalgebra of $F(S)$ ), is a pullback;

(f) the same as (e) but assuming that $S$ is finite.

Proof The implications (a) $\Longrightarrow$ (b), (b) $\Longrightarrow$ (c), (c) $\Longrightarrow$ (d), and (e) $\Longrightarrow$ (f) are trivial. (b) $\Longrightarrow$ (a) follows from the Special Adjoint Functor Theorem (see e.g. Theorem 2 in [20, Chapter V, Section 8]) and the fact that the category $\mathbf{C}$ is co-well-powered. (c) $\Longrightarrow$ (b) is also easy since:

- (b) is true for $\mathbf{C}=$ Sets;

- $\quad$ since the forgetful functor $\mathbf{C} \rightarrow$ Sets preserves reflexive coequalizers, this implies that $f^{*}$ preserves reflexive coequalizers;

- colimits can be calculated via coproducts and reflexive coequalizers, and coproducts can be calculated via filtered colimits and finite coproducts;

- pullback functors of algebraic categories always preserve filtered colimits since those are calculated as in Sets;

- preservation of finite coproducts is equivalent to preservation of binary coproducts and the empty coproduct.

(d) $\Longrightarrow$ (c) follows from the fact that pullback functors between algebraic categories preserve filtered colimits and reflexive coequalizers. (d) $\Longleftrightarrow$ (f) follows from the fact that diagram (2.1) can be identified with the diagram

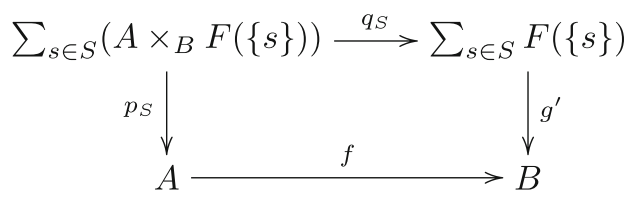


where $g^{\prime}(s)=g(s)$ for $s \in S$, and $q_{S}(a, t)=t$ for $(a, t) \in A \times{ }_{B} F(\{s\})$. Similarly, (e) is equivalent to the infinite version of $(\mathrm{d})$ (that it, to the version of $(\mathrm{d})$ where the finiteness assumption is dropped), while that infinite version of (d) trivially follows from (b).

Corollary 2.2 If $\mathbf{C}$ has no 0-ary operations, then $f: A \rightarrow B$ is exponentiable if and only if the functor $f^{*}$ preserves binary coproducts.

Consider a commutative diagram in $\mathbf{C}$ of the form

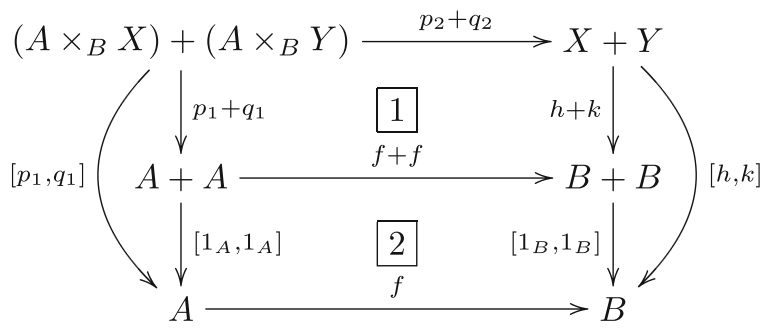

where the $p$ 's and $q$ 's are the appropriate pullback projections. To require the preservation of binary coproducts by $f^{*}$ is to require that the rectangle $\frac{1}{2}$ is always a pullback, while the rectangle 2 is a special case of it. Therefore we have:

Proposition 2.3 The functor $f^{*}$ preserves binary coproducts if and only if the rectangles 1 and 2 in (2.2) are pullbacks for all $h: X \rightarrow B$ and $k: X \rightarrow B$.

\section{The Taut Monad Case}

Let us recall from [4]:

\section{Definitions 3.1}

(1) $T$ is taut (in the sense of E. Manes [21]) if it preserves pullbacks of arbitrary maps along injections, or, equivalently, $F$ preserves such pullbacks;

(2) $f: A \rightarrow B$ is a discrete fibration if the diagram

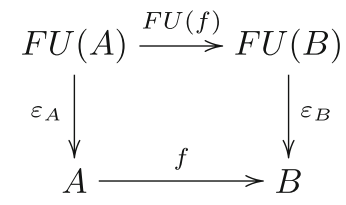

is a pullback;

(3) $f: A \rightarrow B$ is étale if it is a pullback stable discrete fibration.

Theorem 3.3 below is a finitary-taut counterpart of the first part of Theorem 5.5 of [4]. Proving it, we will use the following obvious lemma:

Lemma 3.2 If $f: A \rightarrow B$ is a discrete fibration, and $g: S \rightarrow U(B)$ any map, then the morphism

$$
\varepsilon_{A} \times 1_{F(S)}: F U(A) \times{ }_{F U(B)} F(S) \rightarrow A \times{ }_{B} F(S)
$$


is an isomorphism.

Theorem 3.3 If $T$ is a (finitary) taut monad, and $f: A \rightarrow B$ is étale, then $f: A \rightarrow B$ is exponentiable.

Proof We can assume that the monad $T$ is non-trivial (see Remark 3.4 below). As follows from Theorem 2.1(a) $\Longleftrightarrow$ (e), it suffices to show that $\sum_{s \in S}\left(A \times{ }_{B} F(\{s\})\right)$ and $A \times{ }_{B} F(S)$ are canonically isomorphic to each other for every map $g: S \rightarrow U(B)$. Indeed, there are canonical isomorphisms

$$
\sum_{s \in S}\left(A \times_{B} F(\{s\})\right) \stackrel{(1)}{\cong} \sum_{s \in S}\left(F U(A) \times_{F U(B)} F(\{s\})\right) \stackrel{(2)}{\cong} \sum_{s \in S} F\left(U(A) \times_{U(B)}\{s\}\right)
$$

$\stackrel{(3)}{\cong} F\left(U(A) \times_{U(B)} S\right) \stackrel{(4)}{\cong} F\left(U\left(A \times_{B} F(S)\right) \times_{U F(S)} S\right) \stackrel{(5)}{\cong} F U\left(A \times_{B} F(S)\right) \times_{F U F(S)} F(S)$

$$
\stackrel{(6)}{\cong}\left(A \times_{B} F(S)\right) \times_{F(S)} F(S) \stackrel{(7)}{\cong} A \times_{B} F(S) .
$$

where, assuming that $S$ is non-empty, the reasons for these isomorphisms to hold are:

(1) Lemma 3.2 (applied to the composite $\{s\} \rightarrow S \rightarrow U(B)$ instead of $g$ );

(2) $T$ is taut and the map $\{s\} \rightarrow U(B)$ is injective;

(3) $F$ being a left adjoint preserves coproducts;

(4) $U$ being a right adjoint preserves pullbacks;

(5) $T$ is taut and $\eta_{S}: S \rightarrow U F(S)$ is injective;

(6) Lemma 3.2 (applied to the projection $A \times{ }_{B} F(S) \rightarrow F(S)$ instead of $f$; that projection is a discrete fibration since $f$ is étale);

(7) is obvious.

When $S$ is empty, the isomorphisms (1) and (2) are trivial, while the other isomorphisms hold for the same reasons as for non-empty $S$.

Remark 3.4 When $T$ is any of the two trivial monads, only one of which is taut, every morphism of $T$-algebras is trivially exponentiable.

\section{Only Isomorphisms are Exponentiable in Ideal Determined Varieties}

As shown by H. P. Gumm and A. Ursini [10] a variety of universal algebras is ideal determined (which is the same BIT in the sense A. Ursini [22, 23]) if and only if it is subtractive (this term was later introduced in [24]) and 0 -regular in the sense of K. Fichtner [7]. We recall the latter two terms:

\section{Definitions 4.1}

(1) $\mathbf{C}$ is subtractive when it admits a constant term 0 and a binary term $s$ satisfying the identities

$$
s(x, 0)=x, s(x, x)=0 ;
$$

(2) $\mathbf{C}$ is 0 -regular, where 0 is a fixed constant term in the theory of $\mathbf{C}$, when every congruence on every algebra $A$ in $\mathbf{C}$ is completely determined by ( $A$ and) its class of 0 . 
In this section we will, however, consider only the case of pointed $\mathbf{C}$, where a constant 0 is not only fixed, but it forms the unique 1-element subalgebra in any algebra. As mentioned in [14], it is obvious that a pointed variety is 0-regular if only if every regular epimorphism in it is normal (=the cokernel of its kernel); accordingly we shall call such varieties normal, which also agrees with Z. Janelidze [16], who calls a pointed regular category normal if its regular epimorphisms are normal. A normal variety can equivalently be defined as a pointed variety in which every morphism with zero kernel is a monomorphism.

Theorem 4.2 Let $\mathbf{C}$ be a pointed variety and let $f: A \rightarrow B$ be exponentiable in $\mathbf{C}$. Then:

(a) $f$ has zero kernel;

(b) if $\mathbf{C}$ is normal, then $f$ is a monomorphism;

(c) if $\mathbf{C}$ is subtractive, then $f$ is a regular epimorphism (=surjective);

(d) if $\mathbf{C}$ is ideal determined, then $f$ is an isomorphism.

Proof (a) follows from the fact that $f^{*}$ must preserve initial object, and (b) follows from (a). (d) follows from (b) and (c), and so we only need to prove (c). For that, consider the square 2 in diagram (2.2), which must be a pullback diagram. In that diagram, for each $b \in B$, we have

$$
f(0)=0=s(b, b)=\left[1_{B}, 1_{B}\right]\left(s\left(\iota_{1}(b), \iota_{2}(b)\right)\right),
$$

where $s$ is as in (4.1) and $\iota_{1}$ and $\iota_{2}$ are the coproduct injections $B \rightarrow B+B$. Therefore there exists (a unique) $t \in A+A$ with $\left[1_{A}, 1_{A}\right](t)=0$ and $(f+f)(t)=s\left(\iota_{1}(b), \iota_{2}(b)\right)$. Using this element $t$ and denoting the zero endomorphisms of $A$ and of $B$ by $0_{A}$ and $0_{B}$, respectively, we calculate

$$
b=s(b, 0)=\left[1_{B}, 0_{B}\right]\left(s\left(\iota_{1}(b), \iota_{2}(b)\right)\right)=\left[1_{B}, 0_{B}\right](f+f)(t)=f\left[1_{A}, 0_{A}\right](t),
$$

which shows that $b$ belongs to the image of $f$. That is, $f$ is surjective, as desired.

Remarks 4.3 Let us assume that $\mathbf{C}$ is an arbitrary pointed category with finite limits and finite coproducts instead of being just a variety of algebras. Theorem 4.2 extends to this categorical context as follows. If $f: A \rightarrow B$ is exponentiable, or, more generally, $f^{*}$ preserves finite coproducts, then:

(a) $f$ has zero kernel.

(b) If all morphisms in $\mathbf{C}$ with zero kernels are monomorphisms, then $f$ is a monomorphism. In particular, this is the case when $\mathbf{C}$ is normal in the sense of [16].

(c) If $\mathbf{C}$ is subtractive in the sense of [15] and regular, then $f$ is a regular epimorphism. In order to extend our proof above to this context it is convenient to use the following result due to D. Bourn and Z. Janelidze [3, Theorem 5.1] (implicitly also present in Section 5 of [2]): a pointed regular category is subtractive if and only if, for every object $A$ in it, the composite

$$
\operatorname{Ker}\left(\left[1_{A}, 1_{A}\right]\right) \stackrel{\operatorname{ker}\left(\left[1_{A}, 1_{A}\right]\right)}{\longrightarrow} A+A \stackrel{\left[1_{A}, 0_{A}\right]}{\longrightarrow} A
$$


is a regular epimorphism. Indeed, consider the commutative diagram

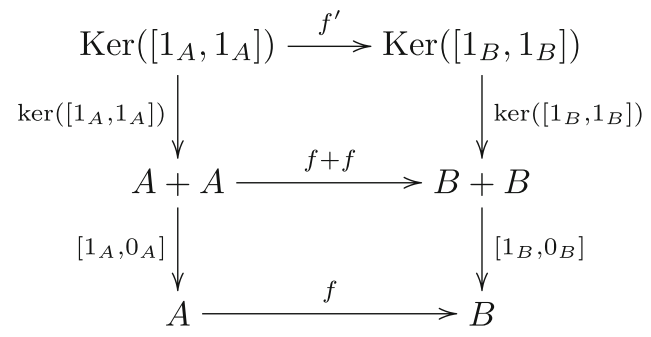

Since the square 2 of diagram (2.2) must be a pullback, $f^{\prime}$ is an isomorphism. Then, since, by Theorem 5.1 of [3], the vertical composites are regular epimorphisms, it follows that so is $f$.

(d) When $\mathbf{C}$ is ideal determined in the sense of [13], it is subtractive and normal, which now implies $f$ is an isomorphism. The normality is in fact a part of the definition of "ideal determined" while subtractivity is a consequence of it, as follows from the results of [8]. In particular, $f$ is an isomorphism whenever $\mathbf{C}$ is semi-abelian in the sense of [12]; this fact, proved a long time ago in a preliminary version of the present paper, was mentioned by J. R. A. Gray [9] with a reference to [4], which we thought then would include the present paper.

\section{Exponentiability of Semimodule Homomorphisms}

Let us use now an enriched-categorical context, where $\mathbf{C}$ is supposed to be a category with finite limits enriched in the category of commutative monoids.

When $\mathbf{C}$ is a variety of algebras this forces $\mathbf{C}$ to be the variety of $S$-semimodules for some semiring $S$ (with 1). This fact is well known and usually considered as "folklore", but it follows from the results of B. Csákány [6] and was clearly formulated and proved by J. S. Johnson and E. G. Manes [17]; we thank Stephen Lack for giving us these references.

That is, the varieties of algebras to which the results of this section apply are exactly the varieties of semimodules. On the other hand, having finite limits in $\mathbf{C}$, to say that $\mathbf{C}$ is enriched in the category of commutative monoids is the same as to say that $\mathbf{C}$ has a zero object and, for every $A$ and $B$ in $\mathbf{C}$, the canonical morphism

$$
\left[\begin{array}{ll}
1 & 0 \\
0 & 1
\end{array}\right]: A+B \rightarrow A \times B
$$

is an isomorphism (this classical result goes back to S. Mac Lane [19]).

The morphism (5.1) being always an isomorphism immediately makes the square 1 in diagram (2.2) a pullback, and so in this case we obtain the following simplified version of Proposition 2.3:

Proposition 5.1 The functor $f^{*}$ preserves binary coproducts if and only if the diagram

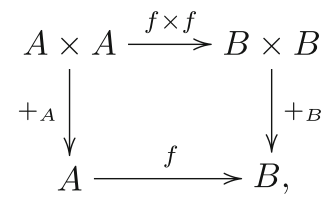


in which $+_{A}=\left[1_{A}, 1_{A}\right]\left[\begin{array}{ll}1 & 0 \\ 0 & 1\end{array}\right]^{-1}$ is the 'internal addition' on $A$ and $+_{B}$ is defined similarly, is a pullback.

We are also interested in the preservation of the initial object, but Corollary 5.2 below makes it trivial.

The internal addition ${ }_{{ }_{A}}$ involved in (5.2), together with the zero morphism $0_{A}: A \rightarrow$ $A$, makes $A$ an internal commutative monoid, and Proposition 5.1 gives

Corollary 5.2 If the functor $f^{*}$ preserves binary coproducts, then $f$ is a monomorphism. In particular $f$ has zero kernel, which implies that $f^{*}$ preserves the initial object.

Proof Let $\left(A \times_{B} A, \pi_{1}, \pi_{2}\right)$ be the kernel pair of $f$; to prove that $f$ is a monomorphism is to prove that $\pi_{1}=\pi_{2}$, or, equivalently, that

$$
\left(\left\langle\pi_{1}, \pi_{2}\right\rangle: A \times{ }_{B} A \rightarrow A \times A\right)=\left(\left\langle\pi_{2}, \pi_{1}\right\rangle: A \times{ }_{B} A \rightarrow A \times A\right) .
$$

We have

$$
\begin{aligned}
+_{A}\left\langle\pi_{1}, \pi_{2}\right\rangle=\pi_{1}+\pi_{2} & =\pi_{2}+\pi_{1}=+{ }_{A}\left\langle\pi_{2}, \pi_{1}\right\rangle, \\
(f \times f)\left\langle\pi_{1}, \pi_{2}\right\rangle=\left\langle f \pi_{1}, f \pi_{2}\right\rangle & =\left\langle f \pi_{2}, f \pi_{1}\right\rangle=(f \times f)\left\langle\pi_{2}, \pi_{1}\right\rangle,
\end{aligned}
$$

which implies $\left\langle\pi_{1}, \pi_{2}\right\rangle=\left\langle\pi_{2}, \pi_{1}\right\rangle$ since diagram (5.2) is a pullback.

Now we are ready to give a complete characterization of exponentiable morphisms of semimodules:

Theorem 5.3 Let $S$ be a semiring and $\mathbf{C}$ be the category of $S$-semimodules. The conditions $(a)-(g)$ below on a morphism $f: A \rightarrow B$ in $\mathbf{C}$ satisfy the implications

$$
(a) \Longleftrightarrow(b) \Longleftrightarrow(c) \Longleftrightarrow(d) \Longleftrightarrow(e) \Longleftrightarrow(f) \Longleftrightarrow(g) \text {. }
$$

Moreover, if $S$ is a quotient semiring of the semiring of natural numbers, then all these conditions are equivalent to each other.

(a) $f$ is exponentiable;

(b) $f^{*}$ preserves binary coproducts;

(c) diagram (5.2) is a pullback;

(d) $f$ is injective and, whenever $f(a)=b_{1}+b_{2}$ in $B$, there exist (uniquely determined) $a_{1}$ and $a_{2}$ in $A$ with $f\left(a_{1}\right)=b_{1}, f\left(a_{2}\right)=b_{2}$, and $a=a_{1}+a_{2}$.

(e) $f$ is injective and, whenever $f(a)=s_{1} b_{1}+\cdots+s_{n} b_{n}$ in $B$ for some natural number $n$ and elements $s_{1}, \ldots, s_{n}$ of $S$, there exist (uniquely determined) $a_{1}, \ldots, a_{n}$ in A with

$$
f\left(a_{1}\right)=b_{1}, \ldots, f\left(a_{n}\right)=b_{n}, \text { and } a=s_{1} a_{1}+\cdots+s_{n} a_{n} ;
$$

(f) $f$ is a discrete fibration;

(g) $f$ is étale.

Proof (a) $\Longleftrightarrow$ (b) follows from Theorem 2.1(a) $\Longleftrightarrow$ (c) and Corollary 5.2. (b) $\Longleftrightarrow$ (c) is nothing but (a special case of) Proposition 5.1. (c) $\Longleftrightarrow$ (d), (e) $\Longrightarrow$ (d), (e) $\Longleftrightarrow$ (f), and $(\mathrm{g}) \Longrightarrow(\mathrm{f})$ are obvious. The remaining implication $(\mathrm{f}) \Longrightarrow(\mathrm{g})$ follows from (e) $\Longleftrightarrow$ (f) and the fact that the class of maps satisfying (e) is (easily seen to be) pullback stable. When $S$ is a quotient semiring of the semiring of natural numbers, we can assume that $s_{1}, \ldots, s_{n}$ 
in (e) are natural numbers and prove $(\mathrm{d}) \Longrightarrow$ (e) by induction on $n$, having in mind that $f$ is injective.

\section{Remarks 5.4}

(a) Every étale morphism of $S$-semimodules is exponentiable. Concerning this fact we can now say:

- It follows from Theorem 5.5 of [4] if we assume that the free $S$-semimodule monad satisfies a version of Beck-Chevalley Condition (see [4] for details), which is analyzed in terms of $S$ in [5].

- It follows from our Theorem 3.3 under a much weaker assumption that the free $S$-semimodule monad it taut; as mentioned in [5], that monad is taut if and only if no non-zero element in $S$ has an additive inverse.

- It is implication $(\mathrm{g}) \Longrightarrow$ (a) of our Theorem 5.3, which is proved above under no additional assumptions at all.

However, the three proofs are surprisingly different from each other.

(b) Among the cases when $S$ is a quotient semiring of the semiring of natural numbers (as in the second assertion of Theorem 5.3), let us mention the following three:

- $\quad S$ is the semiring of natural numbers; in this case $\mathbf{C}$ is the category of commutative monoids.

- $S=\{0,1\}$ with $1+1=1$; in this case $\mathbf{C}$ is the category of semilattices.

- $\quad S$ is a ring; this makes $\mathbf{C}$ an abelian category, and implies that any of the conditions (a) $-(\mathrm{g})$ of Theorem 5.3 holds if and only if $f$ is an isomorphism - however, the fact that all exponentiable morphisms in an abelian category are isomorphisms also follows from Remark 4.3(d) of course.

(c) To see that the implication (d) $\Longrightarrow$ (e) in Theorem 5.3 does not hold in general, consider the following simple example. Let $S$ be the monoid semiring $\mathbb{N}[M]$ over the semiring $\mathbb{N}$ of natural numbers with $M=\{1, x\}$ and $x^{2}=x$. Let $B=\mathbb{N}[M]$ as $\mathbb{N}[M]$ semimodule, $A=x B$, and let $f: A \rightarrow B$ be the inclusion map. Then $f$ obviously satisfies (d), but it does not satisfy (e) since $x=x \cdot 1$ in $A$ while there is no $a$ in $A$ with $f(a)=1$.

Acknowledgments Research partially supported by Centro de Matemática da Universidade de Coimbra - UID/MAT/00324/2013, by Centro de Investigação e Desenvolvimento em Matemática e Aplicações da Universidade de Aveiro/FCT - UID/MAT/04106/2013, funded by the Portuguese Government through FCT/MEC and co-funded by the European Regional Development Fund through the Partnership Agreement PT2020, by South African NRF, and by Georgian Shota Rustaveli National Science Foundation Grant DI/18/5-113/13.

\section{References}

1. Borceux, F., Janelidze, G., Kelly, G.M.: On the representability of actions in a semi-abelian category. Theory Appl. Categ. 14(11), 244-286 (2005)

2. Bourn, D., Janelidze, Z.: Approximate Mal'tsev operations. Theory Appl. Categ. 21, 152-171 (2008)

3. Bourn, D., Janelidze, Z.: Subtractive categories and extended subtractions. Appl. Categ. Struct. 17, 317343 (2009)

4. Clementino, M.M., Hofmann, D., Janelidze, G.: On exponentiability of étale algebraic homomorphisms. J. Pure Appl. Algebra 217, 1195-1207 (2013) 
5. Clementino, M.M., Hofmann, D., Janelidze, G.: The monads of classical algebra are seldom weakly cartesian. J. Homotopy Relat. Struct. 9(1), 175-197 (2014)

6. Csákány, B.: Primitive classes of algebras which are equivalent to classes of semi-modules and modules (in Russian). Acta Sci. Math. (Szeged) 24, 157-164 (1963)

7. Fichtner, K.: Eine Bemerkungüber Mannigfaltigkeiten universeller Algebren mit Idealen. Monatsb. Deutsch. Akad. Wiss. Berlin 12, 21-25 (1970)

8. Gran, M., Janelidze, Z., Rodelo, D., Ursini, A.: Symmetry of regular diamonds, the Goursat property, and subtractivity. Theory Appl. Categ. 27(6), 80-96 (2012)

9. Gray, J.R.A.: Algebraic exponentiation in general categories. Appl. Categ. Struct. 20(6), 543-567 (2012)

10. Gumm, H.P., Ursini, A.: Ideals in universal algebras. Algebra Univ. 19, 45-54 (1984)

11. Higgins, P.J.: Groups with multiple operators. Proc. London Math. Soc. (3) 6, 366-416 (1956)

12. Janelidze, G., Márki, L., Tholen, W.: Semi-abelian categories. J. Pure Appl. Algebra 168, 367-386 (2002)

13. Janelidze, G., Márki, L., Tholen, W., Ursini, A.: Ideal-determined categories. Cah. Topol. Géom. Différ. Catég. 51(2), 115-125 (2010)

14. Janelidze, G., Márki, L., Ursini, A.: Ideals and clots in universal algebra and in semi-abelian categories. J. Algebra 307(1), 191-208 (2007)

15. Janelidze, Z.: Subtractive categories. Appl. Categ. Struct. 13, 343-350 (2005)

16. Janelidze, Z.: The pointed subobject functor, $3 \times 3$ lemmas, and subtractivity of spans. Theory Appl. Categ. 23(11), 221-242 (2010)

17. Johnson, J.S., Manes, E.G.: On modules over a semiring. J. Algebra 15, 57-67 (1970)

18. Johnstone, P.T.: Collapsed toposes and cartesian closed varieties. J. Algebra 129, 446-480 (1990)

19. Lane, S.M.: Duality for groups. Bull. Amer. Math. Soc. 56, 485-516 (1950)

20. Mac Lane, S.: Categories for the working mathematician, graduate texts in mathematics. SpringerVerlag, New York-Berlin (1971)

21. Manes, E., Monads, T.: T0-spaces. Theoret. Comput. Sci. 275, 79-109 (2002)

22. Ursini, A.: Sulle varietà di algebre con una buona teoria degli ideali. Boll. Un. Mat. Ital. (4) 6, 90-95 (1972)

23. Ursini, A.: Osservazioni sulle varietà. B I T Boll. Un. Mat. Ital. (4) 7, 205-211 (1973)

24. Ursini, A.: On subtractive varieties I. Algebra Univ. 31, 204-222 (1994) 